\title{
IMPORTÂNCIA DAS CORES NO CRESCIMENTO, BEM-ESTAR E REPRODUÇÃO DE PEIXES
}

Fernanda Keley Silva Pereira Navarro ${ }^{1}$ Rodrigo Diana Navarro²

NAVARRO, F. K. S. P.; NAVARRO, R. D. Importância das cores no crescimento, bem-estar e reprodução de peixes. Arq. Ciênc. Vet. Zool. UNIPAR, Umuarama, v. 20, n. 1, p. 45-48, jan./mar. 2017.

RESUMO: O Brasil possui alto potencial para piscicultura devido às suas condições ambientais favoráveis à elevada diversidade de espécies nativas com potencial para corte e para ornamentação e à vasta disponibilidade de recursos hídricos. Os estímulos ambientais são cruciais na modulação do ciclo de vida dos animais e, dessa forma, as modificações inerentes a cor do ambiente pode afetar o comportamento e o desenvolvimento dos peixes. Poucos estudos têm sido dedicados ao entendimento dos efeitos do fundo ou cor de luz sobre a biologia dos peixes. Objetivou-se fazer uma revisão bibliográfica sobre os efeitos das cores no bem-estar, na reprodução e no crescimento dos peixes. A luminosidade, por exemplo, é uma condição ambiental essencial para a sobrevivência dos peixes. A luz também é o principal fator responsável pelo controle do ritmo biológico dos animais. A resposta ao estresse corresponde a uma série de alterações fisiológicas. Os efeitos são divididos em primários, secundários e terciários. A manipulação deste sincronizador ambiente pode auxiliar na produção e na qualidade do pescado dentro da área de piscicultura. O efeito das cores sobre o desempenho produtivo e reprodutivo de peixes dependerá dentre outros fatores, da espécie a ser estudada, do sexo e do estágio de desenvolvimento dessa espécie, e é de suma importância para melhor o bem-estar dos animais e obter maiores rendimentos no setor de piscicultura.

PALAVRAS-CHAVE: Condições ambientais. Comportamento. Desempenho.

\section{IMPORTANCE OF COLORS ON GROWTH, WELFARE AND REPRODUCTION OF FISH}

ABSTRACT: Brazil has a high potential for fish farming due to its favorable environmental conditions to a high diversity of native species with potential for food consumption and for decoration, as well as its vast availability of water resources. Environmental stimuli are crucial in modulating the life cycle of animals, and therefore the inherent color changes of the environment can affect the behavior and development of fish. Few studies have been devoted to understanding the effects of background or light color on fish biology. The purpose of this paper was to make a literature review on the theme of color effects on fish welfare, reproduction and growth. Brightness, for instance, is an essential environmental condition for fish survival. Light is also one of the main factors responsible for controlling the biological rhythm of those animals. Stress response corresponds to a series of physiological changes, with their effects being divided into primary, secondary and tertiary. The manipulation of this environmental synchronizer can aid in the production and quality of fish within the fish farming area. The effect of colors on the productive and reproductive performance of fish species under study depends, among other factors, on the sex and development stage of this species, and is of paramount importance for better animal welfare and higher yields in the fish farming sector.

KEYWORDS: Behavior. Environmental conditions. Performance.

\section{IMPORTANCIA DE LOS COLORES EN EL CRECIMIENTO, BIENESTAR Y REPRODUCCIÓN DE PECES}

RESUMEN: Brasil tiene un gran potencial para la piscicultura debido a sus favorables condiciones ambientales, la alta diversidad de especies nativas con potencial para corte y adorno, y la gran disponibilidad de recursos hídricos. Los estímulos ambientales son cruciales en la modulación del ciclo de vida de los animales y, de esa forma, las modificaciones inherentes al color del ambiente puede afectar el comportamiento y el desarrollo de los peces. Pocos estudios se han dedicado a la comprensión de los efectos de fondo o color de luz sobre la biología de los peces. Se ha buscado una revisión bibliográfica sobre los efectos de los colores en el bienestar, en la reproducción y en el crecimiento de los peces. La luminosidad, por ejemplo, es una condición ambiental esencial para la supervivencia de los peces. La luz también es el principal factor responsable por el control del ritmo biológico de los animales. La respuesta al estrés corresponde a una serie de cambios fisiológicos. Los efectos se dividen en primarios, secundarios y terciarios. El manejo de este entorno sincronizador puede ayudar en la producción y la calidad del pez dentro del área de la acuicultura. El efecto de los colores sobre el desempeño productivo y reproductivo de peces dependerá entre otros factores, de la especie a ser estudiada, del sexo y de la etapa de desarrollo de esa especie, y es de suma importancia para mejorar el bienestar de los animales y obtener mayor rendimiento en el sector de la acuicultura. PALABRAS CLAVE: Condiciones ambientales. Comportamiento. Rendimiento.

DOI: https://doi.org/10.25110/arqvet.v20i1.2017.6320

${ }^{1}$ Professora de Biologia, Instituto Federal de Educação, Ciência e Tecnologia de Goiás, 72910-733, Águas Lindas de Goiás, GO, Brasil.

${ }^{2}$ Professor de aquicultura, Laboratório de Aquicultura e de Biotecnologia em Organismos Aquáticos, Faculdade de Agronomia e Medicina Veterinária, Universidade de Brasília, Campus Universitário Darcy Ribeiro, 70910-970, Brasília, DF, Brasil. 


\section{Introdução}

$\mathrm{O}$ aumento da demanda mundial por proteína de origem animal e de boa qualidade vem contribuindo para impulsionar a aquicultura brasileira. Desde 1970, esta atividade vem crescendo à taxa média de $9,2 \%$ ao ano e superando a produção de outros animais, que cresce apenas $2,8 \%$, tornando-se, assim, uma das atividades econômicas de maior crescimento (BEZERRA et al., 2008). Inserida nas atividades da aquicultura, a piscicultura vem se concretizando cada vez mais como atividade relevante. Segundo a Organização das Nações Unidas para Alimentação e Agricultura-FAO, a produção mundial de Pescado em 2012 foi de 158 milhões de toneladas, das quais 136,2 milhões de toneladas foram utilizadas no consumo humano. Neste cenário o Brasil ocupa a $12^{\mathrm{a}}$ posição em produção aquícola. Como os estoques pesqueiros estão estagnados por atingirem o limite no seu extrativismo, a aquicultura será a responsável por atender a demanda crescente, sendo hoje responsável por aproximadamente $48,9 \%$ do total do pescado produzido (FAO, 2014). A produção aquícola brasileira vem apresentando crescimento devido à crescente demanda por pescado no mercado interno e ao imenso potencial do país para a atividade que poderá levar o Brasil a se tornar um dos maiores produtores de peixes do mundo.

O Brasil possui alto potencial para piscicultura devido às suas condições ambientais favoráveis à elevada diversidade de espécies nativas com potencial para corte e para ornamentação e à vasta disponibilidade de recursos hídricos. Entre as espécies mais cultivadas, estão as de origem exóticas, como a carpa (Cyprinus carpio), a tilápia (Oreochromis niloticus) e a truta (Oncorhynchus Kiss) (MENDONÇA et al., 2009).

Os estímulos ambientais são cruciais na modulação do ciclo de vida dos animais e, dessa forma, as modificações inerentes ao ambiente podem afetar o comportamento (ADAMO; PARSONS, 2006, NAVARRO et al., 2013; NAVARRO et al., 2014). O efeito das variáveis ambientais sobre o crescimento, sobrevivência, respostas fisiológicas dos peixes, e comportamento têm sido extremamente estudadas. Alguns estudos e investimentos científicos vêm sendo feitos na área de piscicultura a fim de minimizar o estresse dos peixes decorrente de diversos fatores como manejo, estado nutricional, qualidade da água, temperatura, luminosidade, salinidade (BANI et al., 2009). Objetivou-se fazer uma revisão bibliográfica sobre os efeitos das cores no bem-estar, na reprodução e no crescimento dos peixes.

\section{Desenvolvimento}

A resposta ao estresse corresponde a uma série de alterações fisiológicas. Os efeitos são divididos em primários, secundários e terciários. Entre os efeitos primários encontram-se os aumentos de catecolaminas, adrenalina e noradrenalina, e corticosteroides no plasma (NAVARRO et al., 2015).

Nos efeitos secundários, existem os efeitos metabólicos como as alterações na glicemia, no ácido láctico, e no glicogênio hepático e muscular, e os hematológicos, como alterações nos hematócritos e no número de linfócitos, além dos efeitos hidrominerais, como alterações na concentração plasmática de cloro, sódio, potássio, proteínas, e na osmolaridade do plasma. Os efeitos terciários são as quedas no desempenho produtivo e reprodutivo e na resistência às doenças (ADAMANTE, 2005).

Em peixes teleósteos, a elevação plasmática de cortisol e glicose são reconhecidas como as principais respostas ao estresse, sendo amplamente utilizada como indicador dessa resposta (ADAMANTE, 2005).

A luminosidade, por exemplo, é uma condição ambiental essencial para a sobrevivência dos peixes (NAVARRO; NAVARRO, 2012). A luz também é o principal fator responsável pelo controle do ritmo biológico dos animais (VOLPATO; TRAJARO, 2006; ZHDANOVA; REEBS, 2006). A diversidade de repostas a luz entre os peixes pode ser reflexo de adaptações específicas ao seu ambiente, onde a luz pode variar em termos de quantidade (intensidade luminosa), qualidade (espectro de absorção) e periodicidade (duração da luz ou fotoperíodo) (FÁLCON et al., 2010).

Além disso, esta corresponde a um dos diversos estímulos ambientais que, em condições de criação, pode ser facilmente manipulada a fim de alterar aspectos fisiológicos. Muitas atividades e padrões comportamentais em peixes são regulados pela luminosidade, tais como, o crescimento (BISWAS et al., 2006), a taxa de sobrevivência (REYNALTE-TATAJE et al., 2002), a densidade populacional (JUELL; FOSSEIDENGEN, 2004), a eficiência alimentar (WHITE et al., 2005; BISWAS et al., 2006; SHENG et al., 2006), a reprodução (NAVARRO; NAVARRO, 2012; RICK; BAKKER, 2008), a taxa metabólica (BISWAS; ENDO; TAKEUCHI, 2002) e a atividade de natação (ALMAZÁN-RUEDA, SCHRAMA; VERRETH, 2004). Além desses, a luminosidade também afeta o comportamento agonístico, no entanto, os resultados são contraditórios (ALMAZÁN-RUEDA et al., 2005).

Em relação ao espectro de luz, a cor do ambiente pode interferir em aspectos da biologia dos animais, principalmente no que se refere ao seu comportamento (SOARES; HAYASHI; FARIA,2001). Nos peixes diurnos, a visão é a principal modalidade sensorial (LOUKASHKIN; GRANT, 1959).

Poucos estudos têm sido dedicados ao entendimento dos efeitos do fundo ou cor de luz sobre a biologia dos peixes. No entanto, alguns efeitos interessantes têm sido relatados por alguns autores. $\mathrm{O}$ aumento da frequência de confrontos agonísticos em peixes estressados são alguns dos efeitos da cor do ambiente sobre o comportamento dos peixes.A função da interação agressiva varia entre as espécies e depende das condições sociais e ambientais (VILLARS, 1983). Em peixes, os confrontos podem definir o acesso a sítios de alimentação e acasalamento (GONÇALVES-DE-FREITAS; NISHIDA, 1998), a defesa de parceiros (YAMAMOTO et al., 1999) e de prole (FITZGERALD; KEENLEYSIDE, 1978). A importância do comportamento agonístico também é evidente nas espécies de peixes da família Cichlidae cuja organização social é baseada na hierarquia de dominância e territorialidade (BAERENDS; BAERENDS e VAN ROON, 1950).

No estudo de Merighe (2001) com tilápias do Nilo, os peixes mantidos nos ambientes preto e verde apresentaram baixas frequências de confrontos agonísticos, enquanto aqueles submetidos às cores marrom e azul apresentaram as mais 
altas frequências de comportamentos agonísticos, maior atividade locomotora além de maiores níveis de cortisol. Outros estudos realizados com tilápias do Nilo (Oreochromisniloticus) sugeriram que as cores preta, branca, amarela e vermelha devem ser evitadas, pois causam diferentes níveis de estresse ou mudanças significativas no comportamento, enquanto a verde seria indicada como a mais adequada em meios artificiais como tanques de criação ou aquários experimentais, por corresponder àquela do ambiente natural de regiões onde a espécie ocorre e não interferir significativamente em seu comportamento (FANTA, 1995). Volpatto e Barreto (2001) observaram que tilápias do Nilo expostos a ambiente de luz azul não apresentaram aumento dos níveis de cortisol além de mostrarem um melhor bem-estar, ao contrário da luz verde. PAPOUTSOGLOU et al. (2000), por intermédio da quantificação do cortisol plasmático, observaram menor grau de estresse em carpas mantidas em ambientes brancos, comparativamente aos ambientes pretos e verdes.

Howell (1977), citado por Dendrinos, Dewan e Thorpe (1984), relatou crescimento mais rápido e maior sobrevivência de larvas de "turbot", Scophthal musmaximus, alimentadas com Brachionuse criadas em tanques pretos, comparativamente àquelas criadas em tanques brancos. $\mathrm{O}$ efeito da cor do meio sobre a alimentação também foi relatado por Ostrowski (1989), que destaca a cor do tanque de criação como o principal fator sobre as taxas de sobrevivência de larvas de Dourado marinho (Coryphaema hippurus) em cativeiro. Este autor encontrou percentagem de sobrevivência significativamente elevada para tanques pretos, comparativamente aos tanques mais claros ou brancos, recomendando o uso de tanques pretos para sistemas em que rotíferos são usados como alimento.

\section{Considerações Finais}

Esta revisão teve por objetivo mostrar que as cores desempenham um importante papel no crescimento, bem-estar e na reprodução de peixes. A manipulação desse sincronizador ambiente pode auxiliar na produção e na qualidade do pescado dentro da área de piscicultura. $\mathrm{O}$ efeito das cores sobre os desempenhos produtivo e reprodutivo de peixes dependerá dentre outros fatores, da espécie a ser estudada, do sexo e do estágio de desenvolvimento dessa espécie e é de suma importância para melhor bem-estar dos animais e para obtenção de maiores rendimentos no setor de piscicultura.

\section{Referências}

ADAMO, S.A.;PARSONS, N.M. The emergency lifehistory stage and immunity in the cricket, Gryllustexensis. Animal Behaviour, London, v. 72, p. 235-244, 2006.

ADAMANTE. W. B. Estresse de alevinos de Dourado e Mandi sob diferentes densidades e tempos de transporte. (Dissertação de Mestrado). Universidade Federal de Santa Catarina, Florianópolis - SC. 39 p. 2005.

ALMAZÁN-RUEDA, P.; SCHRAMA, J.W.; VERRETH, J. A. J. Behavioural responses under different feeding methods and light regimes of the African catfish (Clarias gariepinus) juveniles. Aquaculture, Amsterdam, v. 231, p. 347-359,
2004.

ALMAZÁN-RUEDA, P. et al. Photoperiod affects growth, behaviour and stress variables in Clarias gariepinus. Journal of Fish Biology, London, v. 67, p. 1029-1039, 2005.

BAERENDS, G. P. AND BAERENDS-VAN ROON, J. M. An Introduction to the Study of the Ethology of Cichlid Fishes, Behaviour (Supplement 1). Leiden:Brill, 1950. $243 \mathrm{p}$.

BANI, A. et al. Effects of different photoperiods on growth, stress and haematological parameters in juvenile great sturgeon Husohuso. Aquaculture Research, Oxford, v. 40, p.1899-1907, 2009.

BEZERRA, K. S. et al. Crescimento e sobrevivência da tilápia chitralada submetida a diferentes fotoperíodos. Pesquisa Agropecuária Brasileira, Rio de Janeiro, v. 43, n. 6, p. 737-743, 2008.

BISWAS, A. K.; ENDO, M.; TAKEUCHI, T. Effect of different photoperiod cycles on metabolic rate and energy loss of both fed and unfed young tilapia Oreochromis niloticus: Part I. Fisheries Science, Tokyo, v. 68, p. 465477, 2002.

BISWAS, A. K. et al. Effect of photoperiod manipulation on the growth performance and stress response of juvenile red sea bream (Pagrus major). Aquaculture, Amsterdam, v. 258, p. 350-356, 2006.

DENDRINOS, P.; DEWAN, S.; THORPE, J. P. Improvement in the feeding efficiency of larval, post larval and juvenile Dover sole (Soleas olea L.) by the use of staining to improve the visibility of Artemiaused as food. Aquaculture, Amsterdam, v. 38, p. 137-144, 1984.

FÁLCON, J. et al. Current knowledge on the melatonin system in teleost fish. General and Comparative Endocrinology, New York, v.165, p. 469-482, 2010.

FANTA, E. Influence of background color on the behavior of the fish Oreochromis niloticus (Cichlidae). Arquivos de Biologia e Tecnologia, Curitiba, v. 38, n. 4, p. 1237-1251, 1995.

FAO. 2014. The State of World Fisheries and Aquaculture 2014. Rome: United Nations Food and Agriculture Organization, 2014. 223 p.

FITZGERALD, G. J.; KEENLEYSIDE, M. H. A. The effects of numerical density of adult fish on reproduction and parental behavior in the convict cichlid Cichlasoma nigrofasciatum (Gunther). Canadian Journal of Zoology, Ottawa, v. 56, p. 1367-1371, 1978.

GONÇALVES-DE-FREITAS, E.; NISHIDA, S.M. Sneaking behaviour of the Nile tilapia. Botetim Técnico do CEPTA, Pirassununga, v. 11, p. 71-79, 1998. 
JUELL, J. E.; FOSSEIDENGEN, J. E. Use of artificial light to control swimming depth and fish density of Atlantic salmon (Salmo salar) in production cages. Aquaculture, Amsterdam, v. 233, p. 269-282. 2004

LOUKASHKIN, A. S.; GRANT, N. Behavior and reactions of the pacific sardine, Sardinops caerulea(Girard), under the influence of white and colored lights and darkness.

Proceedings of the California Academy of Sciences, San Francisco, v. 29, p. 509-548, 1959.

MENDONÇA, et al. Influência do fotoperíodo no desenvolvimento de juvenis de Tambaqui (Colossoma macropomum). Archivo de Zootecnia, Córdoba, v. 58, p. 323-331, 2009

MERIGHE, G. K. F. Efeito da cor do ambiente e situações sociais sobre o comportamento e parâmetros fisiológicos de tilápias do Nilo Oreochromisniloticus. 2001. Dissertação (Mestrado) - Pirassununga, FZEA, Universidade de São Paulo, Pirassununga, 2001.

NAVARRO, F. K. S. P.; NAVARRO, R. D. Importância do fotoperíodo no crescimento e na reprodução de peixes. Revista Brasileira Reprodução Animal, Belo Horizonte, v. 36, n. 2, p. 94-99, 2012.

NAVARRO, et al.The effect of different photoperiods on plasma levels of LH and gonadal maturation of female lambari (Astyanax bimaculatus). Pesquisa Agropecuária Brasileira, Rio de Janeiro, v. 48, n. 8, p. 1064-1070, 2013.

NAVARRO et al. Effect of photoperiod stress assessment and locomotor activity of female lambari (Astyanaxbimaculatu). Ciência e Agrotecnologia, Lavras, v. 38, n. 2, p. 173-180, 2014.

NAVARRO, et al. Effects of photoperiod on somatic growth and gonadal development in male nile tilapia. Acta Scientiarum. Technology, Maringá, v. 37, p. 381, 2015.

OSTROWSKI, A. C. Effect of rearing tank background color on early survival of dolphin larvae. The Progressive Fish-Culturist, Washington, v. 51, n. 3, p.161-163, 1989.

PAPOUTSOGLOU, S. E. et al. Effects os background color on growth performances and physiological responses of scaled carp (Cyprinus carpio L.) reared in a closed circulated system. Aquaculture Engineer, London, v. 22, n. 4, p. 309-318, 2000.

REYNALTE-TATAJE, D. et al. Influência do fotoperíodo no crescimento e sobrevivência de pós-larvas de piracanjuba Bryconor bignyanus (Valenciennes, 1849) (Osteichthyes, Characidae). Acta Scientiarum, Maringá, v. 24, p. 439-443, 2002.

RICK, I.P.; BAKKER, T.C.M. UV wavelengths make female three-spined sticklebacks (Gasterosteus aculeatus) more attractive for males. Behavioral Ecology and Sociobiology, v. 62, p. 439-445, 2008.
SHENG, J. et al. Effects of food, temperature and light intensity on the feeding behavior of three-spot juvenile seahorses, Hippocampus trimaculatus Leach. Aquaculture, Amsterdam, v. 256, p. 596-607, 2006.

SOARES, C. M.; HAYASHI, C.; FARIA, A. C. E. A Influência da disponibilidade de presas, do contraste visual e do tamanho das larvas de Pantala sp. (Odonata, Insecta) sobre a predação de Simocephalusserrulatus (Cladocera, Crustacea). Acta Scientiarum, Maringá, v. 23, n. 2, p. $357-$ $362,2001$.

VILLARS, T. A. Hormones and aggressive behavior in teleost fishes. In: SVARE, B. B. Hormones and aggressive behavior. Plenum Press, New York and London, 611p, 1983.

VOLPATO, G. L.; BARRETO, R. E. Environmental blue light prevents stress in the fish Nile tilapia. Brazilian Journal of Medical and Biological Research, São Paulo, v. 34, n. 8, p. 1041-1045, 2001.

VOLPATO, G. L.; TRAJANO, E. Biologicalrhythms. In: VAL, L. A.; VAL, V. M. F. A.; RANDALL, D. J. (Org.). Fish Physiology. Elsevier \& Academic Press, San Diego, p. 101-153, 2006.

WHITE, E.M. et al. Spectral irradiance and foraging efficiency in the guppy, Poecilia reticulata. Animal Behaviour, London, v. 69, p. 519-527, 2005.

YAMAMOTO, M. E. et al.Mate guarding in an Amazonian cichlid, Pterophyllum scalare. Journal of Fish Biology. London, v. 55, p. 888-891, 1999.

ZHDANOVA, I.V.; REEBS, S.G. Circadian Rhythms in Fish. In: SLOMAN, K. A.; WILSON, R. W.; BALSHINE, S. (Org.). Behaviour and physiology of fish. Academic Press, p. 197-22, 2006.

Recebido em: 04.02.2016 Aceito em: 30.05.2017 